\title{
Is there a role for interferon in acute viral hepatitis?
}

\author{
R Esteban
}

\begin{abstract}
Acute hepatitis B is a self limiting disease that resolves spontaneously in $95 \%$ of patients who acquire the infection in adulthood. Patients with acute hepatitis $B$, however, usually take between four and 12 weeks to recover; the personal, social, and sanitary costs associated with this are high. Also, 5-10\% of patients with acute hepatitis $B$ go on to develop chronic liver disease. Hepatitis $C$, by comparison, is an asymptomatic disease associated with a high progression to the chronic stage. At least $50 \%$ of patients with post-transfusion hepatitis $\mathrm{C}$ develop chronic liver disease or cirrhosis. While the efficacy of alpha interferon in chronic hepatitis $B$ and $C$ is now well established, only preliminary evidence for use in the acute phase exists. This paper reviews the preliminary evidence and concludes that, given the high risk of progression to the chronic stage, the use of interferon in acute hepatitis $\mathrm{C}$ should be recommended. (Gut 1993; supplement: S77-S80)
\end{abstract}

Infection with hepatitis B virus (HBV) can result in two different clinical pictures, depending upon the age of infection. In the newborn infected by mother to child transmission, hepatitis B infection is usually asymptomatic, associated with high values of viral replication, and mild histological lesions, and becomes chronic in more than $95 \%$ of cases. The strategy for preventing this type of infection is obviously the sero-vaccination of those born to hepatitis B surface antigen (HBsAg) positive mothers. This is the pattern of HBV infection seen in countries from South East Asia and Africa in which the prevalence of $\mathrm{HBsAg}$ carriers is very high.

In Western countries, HBV infection is mainly acquired during adulthood through sexual or parenteral routes. Infection is often symptomatic, with a clinical picture of acute hepatitis that lasts for five to 12 weeks and eventually resolves spontaneously in $95 \%$ of cases.

Acute hepatitis B is a severe disease and its symptoms are poorly tolerated by patients. This has economic and social implications in terms of the cost of health care and the loss of labour. At present, there is no specific treatment for acute hepatitis B. There is justification for the use of a treatment which would shorten the duration of the illness and prevent the development to the chronic stage. Because of the low occurrence of chronic disease, however, it is difficult to obtain the numbers of patients required to study this aspect adequately.

By comparison, hepatitis $\mathrm{C}$ virus (HCV) infection usually causes an asymptomatic disease which is associated with a high rate of progression to the chronic state. It is widely accepted that at least $50 \%$ of patients with post-transfusion hepatitis $\mathrm{C}$ develop chronic liver disease or cirrhosis. Alpha interferon (IFN) has been used in the treatment of chronic hepatitis $\mathrm{C}$ as it returns alanine aminotransferase (ALT) activities to normal, improves the inflammatory lesions of the liver, and inhibits $\mathrm{HCV}$ replication. Only preliminary evidence exists for the clinical use of interferon alfa in the acute phase of $\mathrm{HCV}$ infection.

\section{Hepatitis B}

Interferon has been shown to be useful in patients with chronic hepatitis $\mathrm{B}$ and acts by inhibiting $\mathrm{HBV}$ replication. About $40 \%$ of those patients treated lose HBV-DNA and $\mathrm{HBeAg}$. It is reasonable, therefore, to test for the possible efficacy in patients with acute hepatitis B.

Tassopoulos and colleagues designed a clinical trial aimed at establishing whether treatment with interferon in acute hepatitis B could modify the severity and duration of symptoms, the clearance of viral markers, or the titre of neutralising antibodies. ${ }^{1}$ The study included 100 patients with acute hepatitis B who were randomised to three groups. Group I consisted of 34 patients who received 3 million units (MU) of interferon alfa-2b three times weekly for three weeks. Group II included 33 patients treated with $10 \mathrm{MU}$ of interferon alfa- $2 b$ three times weekly for three weeks, and group III, with 33 patients, received placebo for the same period of time.

Interferon was well tolerated in all patients, even those with a prolonged prothrombin time or marked jaundice. None of the patients treated with IFN alfa-2b experienced a flare up of the disease or developed encephalopathy. Although the overall clinical, biochemical, and serological outcome was similar in the three groups, patients in group I, who received $3 \mathrm{MU}$ of interferon, showed a clinical improvement in the signs and symptoms of acute hepatitis appreciably earlier than those in the control group. At the end of treatment, ALT activities and bilirubin values were similar between groups, although in group II, that is in patients treated with $10 \mathrm{MU}$ of IFN, ALT activities returned to normal earlier. ${ }^{1}$ There was no 
difference in the clearance rate of $\mathrm{HBsAg}$ between groups, but women in group I eliminated this marker more rapidly than men. HBV-DNA seemed to become negative in Group I earlier than in the other two groups, although there was not a statistically significant difference. In group I, $91.2 \%$ of patients were HBV-DNA positive at onset compared with only $17 \cdot 6 \%$ after two weeks of treatment.

The group that received $10 \mathrm{MU}$ of interferon (Group II) showed higher geometric mean titre $(85 \cdot 1 \mathrm{IU} / \mathrm{l})$ than patients treated with $3 \mathrm{MU}$ $(40 \cdot 7 \mathrm{IU} / \mathrm{l})$ or placebo $(35 \cdot 5 \mathrm{IU} / \mathrm{l}) .^{1}$

In summary, the study by Tassopoulos $e t a l^{1}$ shows that alpha interferon is well tolerated in acute hepatitis B, even in patients with severe disease. Treatment seems to shorten the period of clinical disease and, as a result of the higher titre of anti-HBs achieved in patients treated with $10 \mathrm{MU}$ TIW, might reduce the clinical complications in subjects at risk. The data are promising and deserve further clinical investigation to confirm and expand the results.

\section{Hepatitis C}

$\mathrm{HCV}$ is transmitted mainly by parenteral routes. Infection is often asymptomatic and is associated with a high rate of progression to the chronic stage. Before the discovery of $\mathrm{HCV}$, it was apparent from studies of post-transfusion hepatitis that a high percentage of patients developed chronic liver lesions. Results from eight prospective studies show that half the patients had raised ALT activities one year after the acute hepatitis, $41 \%$ already had chronic active hepatitis, and $20 \%$ had cirrhosis.

HCV infection can eventually lead to the development of hepatocarcinoma. For example, there is the reported case history of a patient who had acute post-transfusion hepatitis, chronic persistent hepatitis, chronic active hepatitis, cirrhosis and, finally, hepatocarcinoma 18 years after transfusion. In our experience, one year after acute posttransfusion hepatitis, $80 \%$ of patients have histological lesions indicative of chronic hepatitis, even in some cases when ALT activities are normal.

In summary, although HCV infection leads to very little symptomatic disease, irreversible chronic lesions are common. It seems reasonable, therefore, to start treatment as early as possible to avoid such an outcome.

\section{Clinical evidence}

Some preliminary studies have reported the use of IFN during the acute phase of $\mathrm{HCV}$ infection to prevent the progression to the chronic stage. There are a few studies with a limited number of patients and different types and doses of interferon, but with follow up periods too short to draw conclusions. For example, Almasio et $a l^{2}$ gave alpha interferon for four weeks to six patients with leukaemia and with acute hepatitis C. Normal ALT activities were achieved in five, although three of them later relapsed. Chemello $e t a l^{3}$ treated nine patients with alpha interferon for 12 weeks. After six months, six patients still had normal ALT activities.

Ohnishi et al ${ }^{4}$ obtained similar results in six patients with acute post-transfusion hepatitis treated with $3 \mathrm{MU}$ three times weekly of human fibroblast $\beta$ interferon for four weeks. ALT activities fell shortly after the start of treatment and remained normal in all patients six and 12 months after treatment finished. The study by Omata and colleagues 5 also deserves emphasis. In a controlled trial of 25 patients, 11 were treated with $\beta$ interferon for four weeks while 14 patients served as controls. One year after treatment, seven of 11 patients $(64 \%)$ had normal ALT activities, compared with only one of the controls (7\%). After this initial trial, three of four patients who did not respond to interferon achieved normal ALT activities after a second course of treatment. After three years of follow up, HCV-RNA by polymerase chain reaction had become negative in 10 of 11 patients treated with $\beta$ interferon, suggesting that interferon normalises ALT activities by inhibiting HCV replication.

In our hospital, we designed a randomised, controlled trial of interferon alfa in 28 patients with acute post-transfusion hepatitis $C ; 619$ were heart surgery patients, four were from general surgery, two from urology, two from gynaecology, and one from internal medicine. Diagnosis of acute post-transfusion non-A, non-B hepatitis was based on standard criteria, and other forms of viral hepatitis were serologically excluded.

The objective of the study was to assess the toleration of the treatment, efficacy in returning ALT activities to normal, and the influence on the histological outcome of the disease. Fifteen patients were randomised to receive $3 \mathrm{MU}$ of IFN alfa-2b three times weekly subcutaneously for 12 weeks, while 13 patients received no treatment, and acted as controls. There were no differences between the groups in terms of age, sex, anti-HCV positivity, incubation period, peak ALT, and the presence of jaundice. Serum samples from all patients were taken weekly during the first month, bi-weekly for the next two months, and then once a month until completing one year of follow up. A liver biopsy was performed at six and 12 months of follow up. Treatment was well tolerated in all patients. Only $40 \%$ of patients had mild flu-like symptoms, and in no case was treatment stopped or the dose of IFN reduced.

Figure 1 shows the serological results of the study. Before treatment, $73 \%$ of patients in the treated group tested positive in the first generation anti-HCV ELISA, and $80 \%$ in the second generation ELISA; all but one patient (93\%) had HCV-RNA detected by polymerase chain reaction using a primer of the $5^{\prime}$ UTR region. All patients in the control group were anti-HCV positive.

At the end of treatment (three months), 11 of 15 patients $(73.3 \%)$ had normal ALT activities, compared with only five of 13 


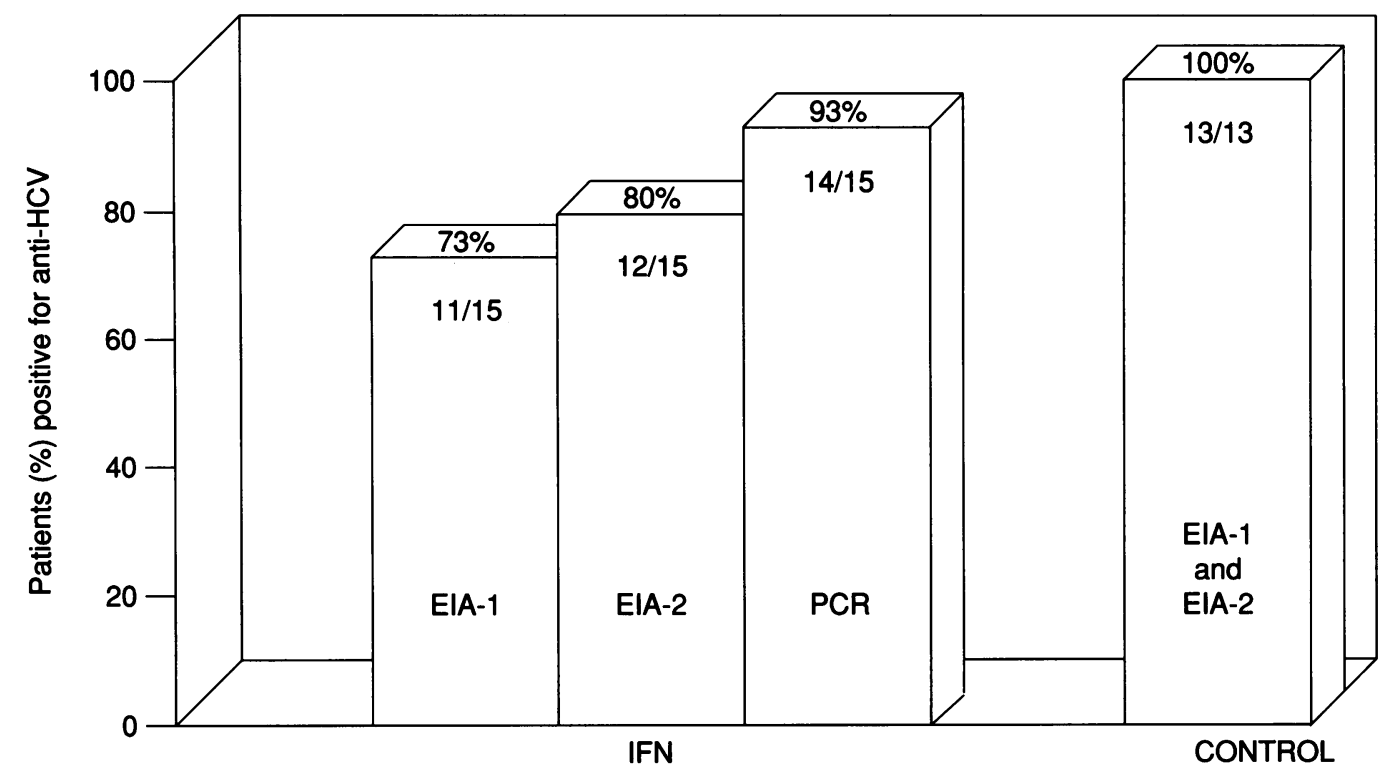

Figure 1 Serological results at the end of treatment (three months).

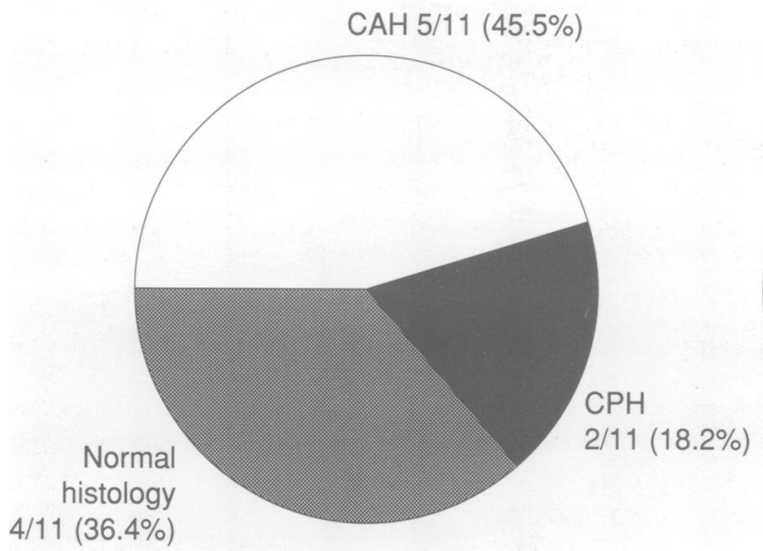

Knodell index $=5$

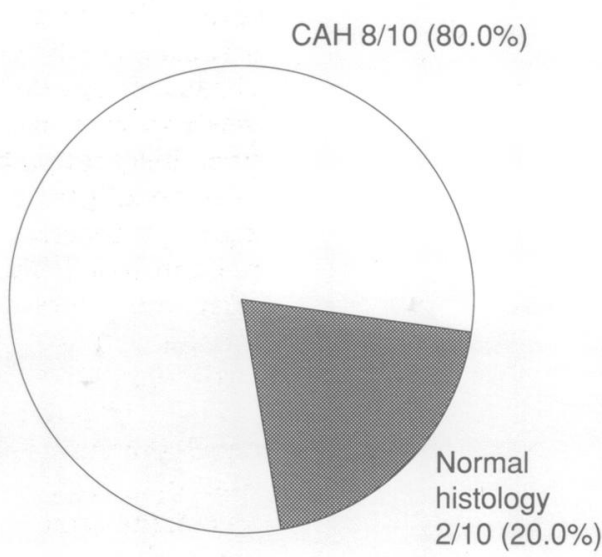

Knodell index $=9.2$

$\mathrm{CPH}=$ Chronic persistent hepatitis

$\mathrm{CAH}=$ Chronic active hepatitis

Figure 2 Results of the liver biopsy performed at 12 months.

controls $(38 \cdot 5 \%)$. The differences between the groups were not statistically significant. At six months, the percentage of patients with normal ALT activities decreased to $46 \%$ in the treated group and $31 \%$ in the control group.

Results of the liver biopsy performed at 12 months are shown in Fig 2. Biopsies were performed in 11 patients treated with IFN and in 10 controls. In the treated group, chronic active hepatitis was found in five patients $(45.5 \%)$, two $(18.2 \%)$ had chronic persistent hepatitis, and four (36.4\%) normal liver on histological examination. Eight of 10 controls $(80 \%)$ had chronic active hepatitis, and the other two patients had almost normal histology. The Knodell index was lower in the group treated with IFN.

We concluded that IFN is well tolerated in patients with acute hepatitis $C$, and is effective in returning ALT activities to normal during the first three months of treatment. This effect disappears during follow up as some patients relapse. Histological lesions at 12 months in the treated group, however, are less severe than the lesions in the control group.

\section{Conclusions}

Although there are only a limited amount of data available for the treatment of acute hepatitis with IFN, results suggest that IFN should be recommended in acute hepatitis C, given the high risk of progression to the chronic stage. Multicentre trials with a longer duration are needed, however, to evaluate definitively the role of IFN in acute HCV infection. 
1 Tassopoulos N, Hadziyannis SJ, Wright GE. A randomized double-blind placebo-controlled trial of alfa interferon in acute type B hepatitis. Hepatology 1989; 10: 575

2 Almasio P, Mirto S, Malleo C, et al. Interferon alfa-2b for acute post-transfusion hepatitis in acute myeloid leukemia. International Symposium on Viral Hepatitis. Houston 1990.

3 Chemello L, Benvegnu L, Bellusi F, Tagger A, Roul A, Alberti A. Pilot study of interferon alpha-2a therapy in preventing chronic evolution of acute hepatitis C. $\mathscr{f}$ Hepatol 1992; 11 (suppl 2): S81.
4 Ohnishi K, Nomura F, Nakano $M$. Interferon therapy for acute posttransfusion non-A, non-B hepatitis: Response with respect to anti-hepatitis $\mathrm{C}$ virus antibody status. $\mathrm{Am}$ with respect to anti-hepatitis $C$ virus antibody status. Am 5 Omata M, Yokosuka O, Takano S, et al. Resolution of acute hepatitis $\mathrm{C}$ after therapy with natural beta interferon. Lancet 1991; 338: 914-5.

6 Viladomiu L, Genesca J, Esteban JI, et al. Interferon-alfa in acute posttransfusion hepatitis C: A randomized, controlled trial. Hepatology 1992; 15: 767-9. 